\title{
HYDROMAGNETIC CONSTRAINTS ON DEEP ZONAL FLOW IN THE GIANT PLANETS ${ }^{1}$
}

\author{
R. L. KIRK AND D. J. STEVENSON \\ Division of Geological and Planetary Sciences, California Institute of Technology \\ Received 1986 September 2; accepted 1986 October 24
}

\begin{abstract}
The observed zonal flows of the giant planets will, if they penetrate below the visible atmosphere, interact significantly with the planetary magnetic field outside the melallized core. The appropriate measure of this interaction is the Chandrasekhar number $Q=H^{2} / 4 \pi \rho v \alpha^{2} \lambda$ ( $H=$ radial component of the magnetic field, $v=$ eddy viscosity, $\lambda=$ magnetic diffusivity, $\alpha^{-1}=$ length scale on which $\lambda$ varies); at depths where $Q \gtrsim 1$, the velocity will be forced to oscillate on a small length scale or decay to zero. We estimate the conductivity due to semiconduction in $\mathrm{H}_{2}$ (Jupiter, Saturn) and ionization in $\mathrm{H}_{2} \mathrm{O}$ (Uranus, Neptune) as a function of depth; the value $\lambda \approx 10^{10} \mathrm{~cm}^{2} \mathrm{~s}^{-1}$ needed for $Q=1$ is readily obtained well outside the metallic core (where $\lambda \approx 10^{2}$ $\mathrm{cm}^{2} \mathrm{~s}^{-1}$ ).

These assertions are quantified by a simple model of the equatorial zonal jet in which the flow is assumed uniform on cylinders concentric with the spin axis, and viscous and magnetic torques on each cylinder are balanced. We solve this "Taylor constraint" simultaneously with the dynamo equation to obtain the velocity and magnetic field in the equatorial plane. With this model we reproduce the widely differing jet widths of Jupiter and Saturn (though not the flow at very high or low latitudes) using $v=2500 \mathrm{~cm}^{2} \mathrm{~s}^{-1}$, consistent with the requirement that viscous dissipation not exceed the specific luminosity. A model Uranian jet consistent with the limited Voyager data can also be constructed, with appropriately smaller $v$, but only if one assumes a two-layer interior. We tentatively predict a wide Neptunian jet.

For Saturn (but not Jupiter or Uranus) the model has a large magnetic Reynolds number where $Q=1$ and hence exhibits substantial axisymmetrization of the field in the equatorial plane. This effect may or may not persist at higher latitudes. The one-dimensional model presented is only a first step. Variation of the velocity and magnetic field parallel to the spin axis must be modeled in order to answer several important questions, including: (1) What is the behavior of flows at high latitudes, whose Taylor cylinders are interrupted by the region with $Q>1$ ? (2) To what extent is differential rotation in the envelope responsible for the spinaxisymmetry of Saturn's magnetic field?
\end{abstract}

Subject headings: hydromagnetics - planets: interiors

\section{INTRODUCTION}

In Earth and other terrestrial planets, we are accustomed to a clear delineation between the highly conducting lowviscosity region (the core), in which hydromagnetics is important, and an outer, very poorly conducting, high-viscosity region (the mantle), in which hydromagnetics is unimportant. Nobody seriously advocates important hydromagnetic effects in plate tectonics, oceanography, or lower atmosphere motions. In the Sun and many other stars, we are equally accustomed to the idea that hydromagnetic effects can be important in the observable atmosphere (e.g., in sunspots). The giant planets do not easily conform to either of these limits. There is little doubt that Jupiter and Saturn possess highly conducting metallic hydrogen cores (Stevenson 1982), but there is also the likelihood that molecular hydrogen regions only a small distance (a few thousand kilometers) below the atmosphere are sufficiently conducting to have significant hydromagnetic effects. The central question is this: To what extent are the observed atmospheric flows (the zonal winds) affected by or even determined by the planetary magnetic field? We offer here only a partial and qualified answer to this question, but an interesting answer nonethless because it suggests a connection between surficial winds, deep-seated flows, and the planetary dynamo.

\footnotetext{
${ }^{1}$ Contributions from the Division of Geological and Planetary Sciences, California Institute of Technology, No. 4337.
}

The ideas are not entirely new. Hide (1965) suggested that the field of Jupiter may be generated near the surface, and Smoluchowski $(1972,1975)$ pointed out the likely semiconducting properties of impure or even pure molecular hydrogen at high pressure and temperature. An attempt has even been made to determine the depth of the field-generating region in Jupiter (Hide and Malin 1979), but the data are insufficient for a convincing result. On the other hand, many workers have assumed that the entire region external to the metallic hydrogen cores of Jupiter and Saturn can be treated as an insulating fluid (i.e., using the Navier-Stokes equation without the Lorentz force). Busse $(1976,1983)$ proposed that the surficial structure of clouds or winds may be directly matched to the columnar convective cells expected deep within rapidly rotating, adiabatic fluid planets. Ingersoll and coworkers, motivated largely by a perceived difficulty in explaining the winds by thin shell meteorology, have pursued the related idea that the observed winds are the surface expression of zonal flows on cylindrical surfaces (Smith et al. 1982; Ingersoll and Pollard 1982; Ingersoll and Miller 1986). This is illustrated in Figure 1. The arguments against confining the winds to a thin shell have become less compelling because of the possibly large role of latent heat effects in the fluid motions (Allison and Stone 1983; Conrath and Gierasch 1984). Although no fully quantitative dynamic theory exists, deep-seated zonal flows still remain an attractive hypothesis, because these planets have bottomless atmospheres and very stable wind patterns. Ironically, the 

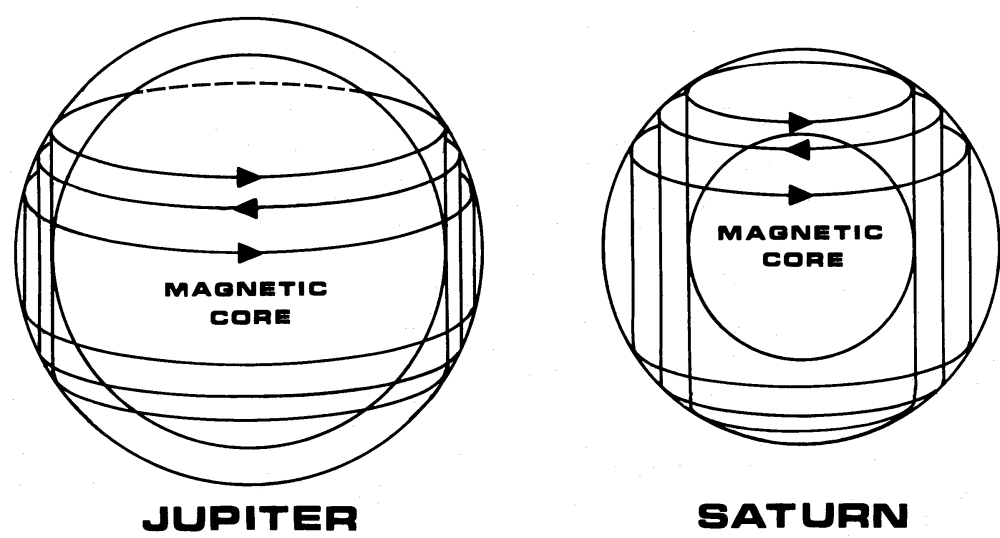

SATURN

Fig. 1.-Schematic representation of deep zonal flow in Jupiter and Saturn. The surface flow extends into the interior on concentric Taylor cylinders but is excluded from a magnetically interacting region at depth.

work reported here provides support for moderately deep seated flows yet invalidates the original views of Busse and Ingersoll, who envisaged columnar or cylindrical flows that completely filled the region external to the metallic hydrogen core.

Our basic ideas are these: A deep zonal flow has a differential rotation that leads to a generation of toroidal field $H_{\phi} \sim$ $R_{m} H_{r}$, where $H_{r}$ is the imposed radial field, $R_{m} \sim v l / \lambda$ is the magnetic Reynolds number, $v$ is the characteristic azimuthal flow, $l$ is some length scale (ill defined, as yet), and $\lambda$ is the magnetic diffusivity. Even for a conductivity ten orders of magnitude less than that of copper at room temperature, $\lambda \sim 10^{12}$ $\mathrm{cm}^{2} \mathrm{~s}^{-1}, R_{m} \sim 1$ for $v \sim 10^{4} \mathrm{~cm} \mathrm{~s}^{-1}$ (typical of Jupiter and Saturn) and $l \sim 10^{8} \mathrm{~cm}$. The toroidal field has an associated poloidal current which, when crossed with the radial field, yields a Lorentz force with an azimuthal component $\sim R_{m} H_{r}^{2} / 4 \pi \rho l$ per unit mass ( $\rho$ is the fluid density). Since there cannot be a net azimuthal torque on a cylinder of fluid in steady flow (Taylor 1963), this force must be balanced by a "viscous" force $\sim v v / l^{2}$, where $v$ is the kinematic eddy viscosity. It follows that we require $H_{r}^{2} l^{2} / 4 \pi \rho v \lambda \sim 1$. This dimensionless number was first introduced by Chandrasekhar (1965, p. 7), although for different reasons. As we go down into the planet, the conductivity increases and $\lambda$ decreases, so this requirement translates into a progressively smaller $l$. In effect, the zonal flow is forced to have large shears. Our thesis is that this requirement imposed by the Chandrasekhar number implies a rapid drop-off in the zonal flow and thereby limits the width of the equatorial jet in giant planets. To put it another way, if these planets did not have magnetic fields, then the observed equatorial jet would extend to much higher latitudes, corresponding to deeper flows. Some aspects of this model were independently developed (but not quantified) by Drobyshevskii $(1979 a, b)$. Here we attempt a quantitative model.

Clearly, the biggest uncertainties lie in the diffusivities $\lambda$ and $v$, which could range over may orders of magnitude. The value of $\lambda$ is computed in $\S \mathrm{II}$, using semiquantitative theories of liquid semiconductors, published band structure calculations of molecular hydrogen, experimental results for the conductivity of water, and published temperature-density structures of giant planets. The value of $v$ might seem to be much more uncertain, because it is not likely to be the very small intrinsic flud value $\left(\sim 10^{-2} \mathrm{~cm}^{2} \mathrm{~s}^{-1}\right)$ but is a crude representation of the nonlinear effects of the flow. However, it is bounded above by the requirements of the first and second laws of thermodynamics: the local viscous dissipation $\sim v(v / l)^{2}$ should not greatly exceed the total planetary thermal energy loss per unit mass $\left(\sim 10^{-6} \mathrm{ergs} \mathrm{g}^{-1} \mathrm{~s}^{-1}\right.$ in Jupiter and Saturn), so $v \lesssim 10^{3} \mathrm{~cm}^{2}$ $\mathbf{s}^{-1}$. In fact, careful scaling arguments (Ingersoll and Pollard 1982) give a value on this order. A Chandrasekhar number of order unity then typically corresponds to the level in the planet at which $\lambda \sim 10^{10} \mathrm{~cm}^{2} \mathrm{~s}^{-1}$. Our thesis is that the spin-aligned cylinder circumscribing the sphere on which this conductivity is obtained must intercept the planetary surface at the latitude corresponding to the outer extremities of the equatorial zonal jet. In this way, we can reproduce the observed width of the jets on Jupiter, Saturn, and possibly Uranus. In $\S$ III, we develop the mathematical theory to support the above heuristic arguments, showing how the Taylor constraint leads to the identification of a Chandrasekhar number. The model is applied to Jupiter and Saturn in $\S$ IV and to Uranus and Neptune in $\S \mathrm{V}$. We end in $\S$ VI with some comments on limitations and possible future work.

\section{THE MAGNETIC DIFFUSIVITY}

In hydromagnetics, it is conventional to characterize the electrical conductivity $\sigma$ in terms of the magnetic diffusivity $\lambda \equiv c^{2} / 4 \pi \sigma$, where $c$ is the speed of light and $\sigma$ is in esu $\left(\mathrm{s}^{-1}\right)$. A resistivity of $1 \mu \Omega \mathrm{cm}$ is equivalent to $\lambda=(250 / \pi) \mathrm{cm}^{2} \mathrm{~s}^{-1}$. Typical values of $\lambda$ are $10^{2}-10^{3} \mathrm{~cm}^{2} \mathrm{~s}^{-1}$ (good metals). $10^{6} \mathrm{~cm}^{2}$ $\mathrm{s}^{-1}$ (good electrolytes), and $\sim 10^{12} \mathrm{~cm}^{2} \mathrm{~s}^{-1}$ for pure or nearly pure germanium at $500 \mathrm{~K}$. Molecular $\mathrm{H}_{2}$ is effectively an insulator at low pressure (band gap $E_{g} \sim 10 \mathrm{eV}$ ), but this gap is believed to diminish progressively as the pressure increases. Although diamond cells (without a hydrogen sample) have now achieved in excess of $4 \mathrm{Mbar}$ (Xu, Mao, and Bell 1986; Goettel et al. 1986) and quantitative experiments on $\mathrm{H}_{2}$ at $\sim 1.5$ Mbar have been reported (Mao, Bell, and Hemley 1985), there are no data on the band gap, except for the inference that the band gap is still finite at the highest pressures attained. The much discussed and anticipated transition to monatomic (alkali metal) hydrogen, conventionally called "metallic hydrogen," probably occurs at much higher pressures still (perhaps $\sim 3-4$ Mbar, see Ross 1985; Min, Jansen, and Freeman 1986) but has no bearing on the issues addressed in this paper.

We rely here on theoretical calculations for the band gap in crystalline $\mathrm{H}_{2}$ (Friedli and Ashcroft 1977; Min, Jansen, and Freeman 1986). These results can be well represented by the 
empirical formula

$$
\begin{aligned}
E_{g} & =32.5 \rho^{2 / 3}\left(x+\epsilon x^{2}\right) \mathrm{eV}, \\
x & \equiv\left(\frac{\rho_{0}}{\rho}\right)^{1 / 3}-1,
\end{aligned}
$$

where $\rho$ is the density in $\mathrm{g} \mathrm{cm}^{-3}, \rho_{0}$ is the density at band closure $\left(0.81 \mathrm{~g} \mathrm{~cm}^{-3}\right.$ for Friedli and Ashcroft, $0.90 \mathrm{~g} \mathrm{~cm}^{-3}$ for Min et al.), and $\epsilon=0.3$ for Friedli and Ashcroft and 0.2 for Min et al. Although there are differences between the two results, they are most striking near band closure, a high-pressure region that does not interest us. A more serious concern is the use of a crystalline calculation to describe a liquid; evidence for liquid semiconductors suggest that the appropriate band gap or mobility gap is probably slightly smaller. Our calculation may therefore be conservative.

In a semiconductor, the fractional occupancy of currentcarrying states is proportional to $\exp \left(-E_{g} / 2 k T\right)$, where $k$ is Boltzmann's constant and $T$ is the temperature. The factor of 2 in the denominator of the exponent is an unavoidable consequence of the law of mass action. It follows that, in general,

$$
\lambda=\lambda_{0} \exp \left(E_{g} / 2 k T\right),
$$

where $\lambda_{0}$ can be a function of density and temperature. Smoluchowski (1975) chose to use the standard crystalline semiconductor result (e.g., Ashcroft and Mermin 1976) in which $\lambda_{0} \sim$ $T^{-3 / 2}$ (no density dependence). We adopt the semiempirical results of the theory of Mott (1971) for liquid semiconductors, according to which $\lambda_{0} \sim 10^{5} \mathrm{~cm}^{2} \mathrm{~s}^{-1}$ and roughly independent of temperature. However, disagreement between the two approaches is an order of magnitude or less at the densities and temperatures of interest. In the low-density limit, $\lambda_{0}$ should eventually approach the prediction of dilute gas theory (Chapman and Cowling 1952), which we estimate to be $\sim 10^{4} \rho^{1 / 3}\left(10^{3} \mathrm{~K} / T\right) \mathrm{cm}^{2} \mathrm{~s}^{-1}$, where $\rho$ is in $\mathrm{g} \mathrm{cm}^{-3}$. We do not use this result, but is approximate consistency with our adopted value of $10^{5} \mathrm{~cm}^{2} \mathrm{~s}^{-1}$ indicates no serious extrapolation difficulties.
The temperature and density profiles within Jupiter and Saturn are obtained from published interior models which differ little in the range of interest (Stevenson and Salpeter 1976; Hubbard and Horedt 1983; Hubbard and Stevenson 1984). At a given fraction of the outer radius, Saturn is much colder than Jupiter, mainly because it has a lower gravitational acceleration but partly because it has a lower specific entropy (i.e., colder atmosphere). However, they are both adiabatic planets, so that the resulting functional dependence of $\lambda$ on fractional planetary radius is the same for the two planets, except for a scale factor. This is exhibited in Figure 2, based on calculations using equations (1) and (2) and the planetary models. The shaded region is an attempt to indicate the combined uncertainties of all the inputs but does not include systematic errors (e.g., the possibility that the mobility gap is systematically smaller than $E_{g}$ as given by eq. [1]).

It is also possible that Figure 2 systematically overestimates the true value of $\lambda$ because of impurities mixed with the hydrogen. However, we doubt that atoms with small ionization energies, such as sodium, are present in a chemically unbound form (as assumed by Smoluchowski 1972). In order of decreasing abundance (approximate mole fractions in brackets), the impurities are expected to be $\mathrm{He}(0.1), \mathrm{H}_{2} \mathrm{O}\left(\sim 10^{-3}\right), \mathrm{CH}_{4}\left(\sim 10^{-3}\right)$, $\mathrm{NH}_{3}\left(\sim 10^{-4}\right), \mathrm{Ne}\left(\sim 10^{-4}\right)$, silicate and iron particles $\left(\sim 10^{-4}\right.$ to $\left.10^{-5}\right)$. None of these appear likely to overwhelm the conductivity at $T \approx 3000 \mathrm{~K}$ (where $\lambda \approx 10^{10} \mathrm{~cm}^{2} \mathrm{~s}^{-1}$ ), the region of most importance for our hydromagnetic effects. Accordingly, we use the results for pure $\mathrm{H}_{2}$.

Our approach to the crude estimates for Uranus and Neptune is different and more closely tied to the data. The conductivity of the envelopes of these planets is controlled by the "ice" component, specifically $\mathrm{H}_{2} \mathrm{O}$ (shock wave experiments suggest that $\mathrm{NH}_{3}$ has about an order of magnitude lower conductivity under similar conditions; Ross, Graboske, and Nellis 1981)-regardless of one's assumptions about the internal structure. At $T \lesssim 2000 \mathrm{~K}$, the "gas" layer of threelayer models (Hubbard and MacFarlane 1980) is too cold to be significantly conductive according to equations (1) and (2), and

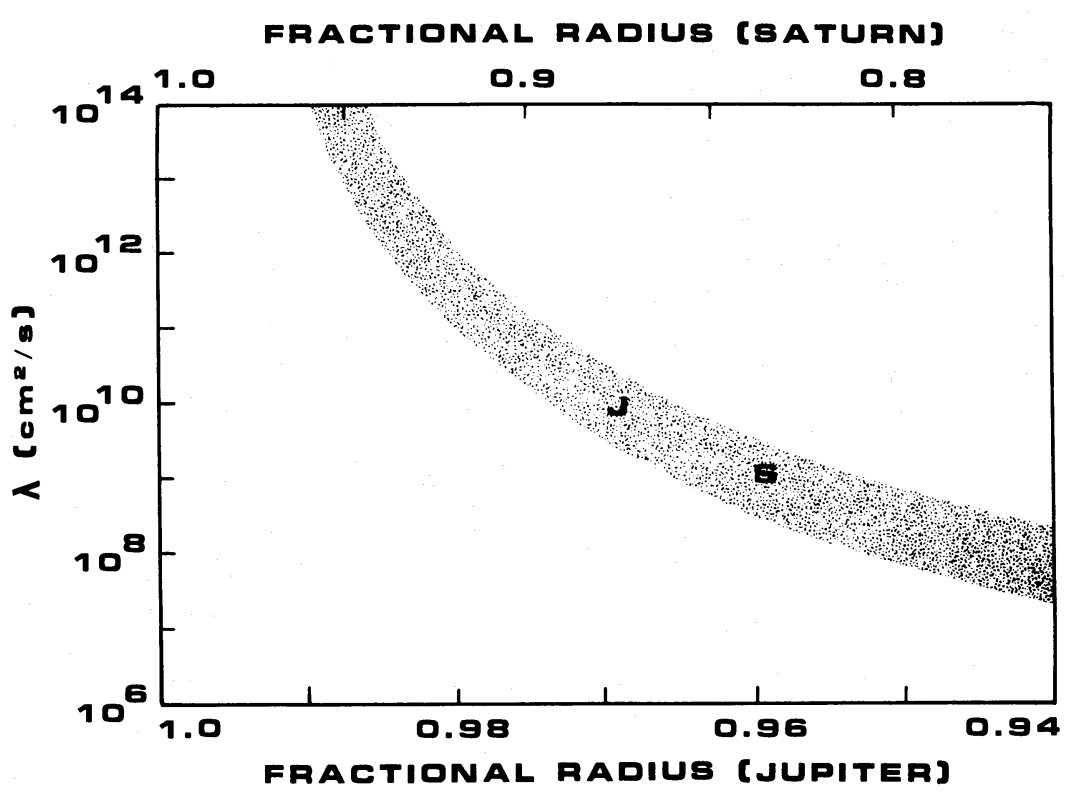

FIG. 2.-Magnetic diffusivity of the Jovian and Saturnian envelopes. The pressure-induced semiconductivity of pure $\mathrm{H}_{2}$ was calculated according to eqs. (1) and (2); the shaded region indicates the uncertainty due to the input data. 
Lorentz forces first become important in the "ice" layer. On the other hand, unpublished two-layer models by one of us (D. J. S.) suggest an ice-to-gas ratio of $\sim 2.5: 1$ in the envelope, and under these circumstances the icy component will dominate the conductivity at any given depth. Accordingly, we compute the conductivity of $\mathrm{H}_{2} \mathrm{O}$ using Holtzapfel's (1968) fit to static and shock wave data at temperatures up to $1000^{\circ} \mathrm{C}$ and pressures to $100 \mathrm{kbar}$. We account for the probable suppression of ionization by dissolved hydrogen/helium in the two-layer model crudely but conveniently by adopting a magnetic diffusivity an order of magnitude greater than that of pure water.

\section{THE TAYLOR CONSTRAINT}

A complete dynamical model of giant planet envelopes which includes hydromagnetic effects is obviously beyond the scope of this paper. We instead present a simple onedimensional model illustrating the effect of inward-increasing conductivity on a dęep-seated pattern of differential rotation. The main assumptions made are; first, that the mean flow is zonal and depends only on cylindrical radius $\left[u=v(s) \hat{e}_{\phi}\right.$ in the $(s, \phi, z)$ cylindrical coordinates of Fig. 3], as guaranteed by the Taylor-Proudman theorem for an inviscid, isentropic, nonconducting medium; second, that the effects of turbulence and convection may be parameterized by a constant eddy viscosity (of either sign as yet); third, that magnetic forces can be expanded about the equatorial plane $z=0$ (where, for given $s$, the conductivity is greatest); and, finally, that the magnetic diffusivity varies exponentially with depth, $\lambda=\lambda_{0} \exp \left[\alpha\left(s^{2}+z^{2}\right)^{1 / 2}\right]$. (In applying our model to the giant planets we linearize the theoretical prediction for $\ln \lambda$ about a point near where $v \rightarrow 0$.)

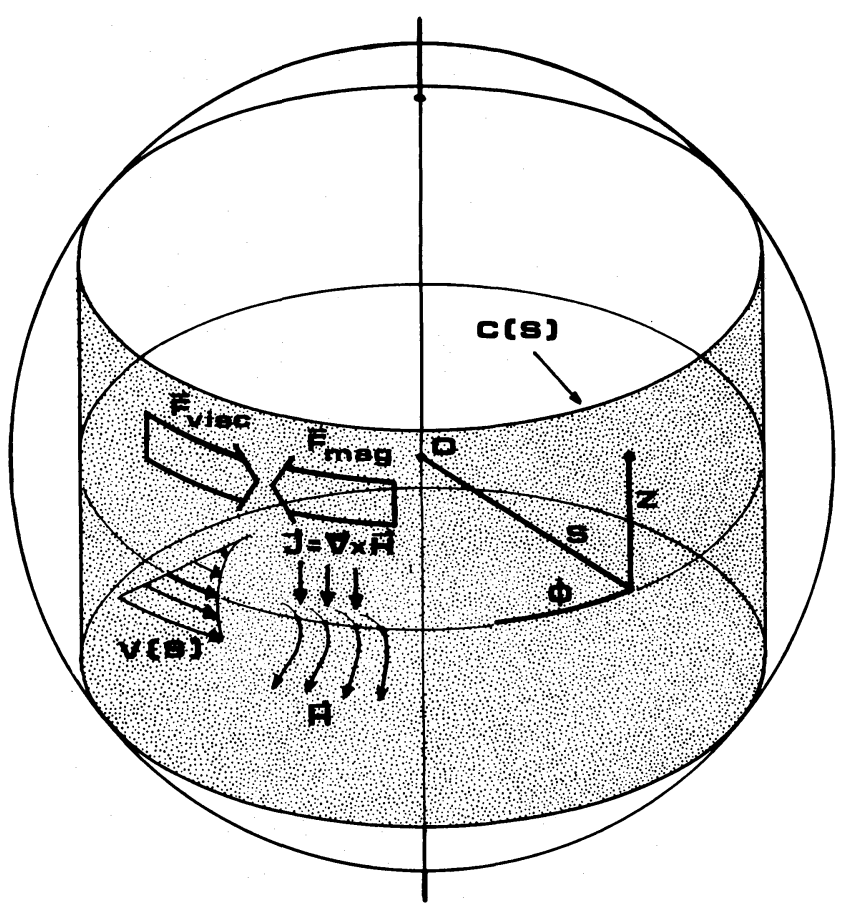

FIG. 3.-The Taylor constraint (eq. [3]) is a balance between the integrated viscous and magnetic torques on a cylindrical surface (Taylor column) $c(s)$ in a differentially rotating planet. The balance is illustrated for a positive eddy viscosity, giving a monotonic zonal velocity $v(s)$. The $(s, \phi, z)$-cylindrical coordinates are replaced with local $(x, y, z)$-Cartesian coordinates for the actual calculations.
For such a system in differential rotation on cylinders, the Taylor constraint (Moffatt 1978) states that the sum of magnetic and viscous torques on a cylindrical surface $c(s)$ vanishes:

$$
\oint_{c(s)}\left[\frac{(v \times H) \times H}{4 \pi \rho} \pm v \nabla^{2} u\right] \cdot \hat{e}_{\phi} s^{2} d \phi d z=0,
$$

where we write the eddy viscosity as $\pm v$ with $v>0$. Local variations of the magnetic torque from its mean value on $c(s)$ will be balanced by pressure gradients which do not enter our analysis.

They Taylor constraint must be supplemented by the dynamo equation for the evolution of the magnetic field, in steady state:

$$
\frac{\partial \boldsymbol{H}}{\partial t}=-\nabla \times(\lambda \nabla \times \boldsymbol{H})+\nabla \times(\boldsymbol{u} \times \boldsymbol{H})=0 .
$$

We also use the solenoidal character of $\boldsymbol{H}$ :

$$
\boldsymbol{\nabla} \cdot \boldsymbol{H}=0 \text {. }
$$

Rather than simplifying equation (3) by formally expanding $\boldsymbol{H}$ about $z=0$, we present here a much simpler derivation in Cartesian coordinates, appropriate to the equatorial plane far from the rotation axis, and the $\partial / \partial z \equiv 0$. The essential features of the problem are more clearly exhibited, and we readily obtain an analytic solution agreeing to lowest order in $|(s / \lambda)(\partial \lambda / \partial s)|^{-1}$ with that obtained by expansion in cylindrical coordinates - provided we measure the velocity with respect to solid-body rotation.

In $(x, y, z)$-coordinates with $\partial / \partial z=0$, the vector equations (4) and (5) hold, but the equivalent of the Taylor constraint is a force balance on a plane of constant $x$ :

$$
\oint\left[\frac{(\nabla \times H) \times \boldsymbol{H}}{4 \pi \rho} \pm v \nabla^{2} u\right] \cdot \hat{e}_{y} d y=0,
$$

where the integral in $y$ is over one cycle of the periodic function $\boldsymbol{H}$. The vertical integral is obviated by the assumption $\partial / \partial z=0$; in applying the solution we assume that the viscous force is constant while the Lorentz term varies on a given cylinder $c(s)$ in proportion to $\sigma$. The result is merely to multiply the first term of equation (6) by a weakly varying function $\delta(s)=\left[\pi s / 2 \alpha\left(R^{2}-s^{2}\right)\right]^{1 / 2}$, since the integral over exponentially varying $\sigma$ is readily performed.

The Lorentz term in the Taylor constraint may be expanded:

$$
\begin{aligned}
{[(\nabla \times \boldsymbol{H}) \times \boldsymbol{H}]_{y} } & =(\boldsymbol{\nabla} \times \boldsymbol{H})_{z} H_{x}-(\nabla \times \boldsymbol{H})_{x} H_{z} \\
& =\left(\frac{\partial H_{y}}{\partial x}-\frac{\partial H_{x}}{\partial y}\right) H_{x}-\frac{\partial H_{z}}{\partial y} H_{z} .
\end{aligned}
$$

The term involving $H_{z}$ is a total differential in $y$ and hence does not contribute to the integral (6). The first term may be rewritten by integrating the dynamo equation (4) to give

$$
\nabla \times H=\frac{u \times H}{\lambda}+\nabla \phi,
$$

where $\phi(x, y)$ is an arbitrary function of integration (fortunately not appearing in the $z$-component of the equation, in which we are interested). These results may be used to simplify the Taylor constraint to yield (with $\boldsymbol{u}=v(x) \hat{e}_{y}$ )

$$
\left[\frac{d^{2}}{d x^{2}} \mp \frac{\left\langle H_{x}\right\rangle^{2} \delta}{4 \pi \rho v \lambda(x)}\right] v=0 \text {. }
$$


As usual, the upper sign corresponds to a positive eddy viscosity, and $\left\langle H_{x}\right\rangle=\left(\oint H_{x}^{2} d y / \oint d y\right)^{1 / 2}$ is the root mean squared value of $H_{x}$. Writing $\boldsymbol{H}=\boldsymbol{H}(x) e^{i k y}+$ complex conjugate, with complex $\boldsymbol{H}(x)$, we have $\left\langle H_{x}\right\rangle=p^{1 / 2}\left|H_{x}\right|\left(p=1\right.$ if $k=0, p=\frac{1}{2}$ otherwise). In cylindrical coordinates $H \sim e^{i m \phi}$, so we identify $k=m / \bar{s}$, with $\bar{s}$ a typical cylindrical radius and $m=0,1$ the harmonics of greatest interest.

With the above assumptions about $\boldsymbol{u}$ and $\boldsymbol{H}$, and $\lambda=\lambda_{0} e^{\alpha x}$, the components of the dynamo equation become

$$
\begin{gathered}
{\left[\lambda\left(\frac{d^{2}}{d x^{2}}-k^{2}\right)-i k v\right] H_{x}=0,} \\
{\left[\lambda\left(\frac{d^{2}}{d x^{2}}+\alpha \frac{d}{d x}-k^{2}\right)\right] H_{y}=\left[i k \alpha \lambda-\frac{d v}{d x}\right] H_{x} .} \\
{\left[\lambda\left(\frac{d^{2}}{d x^{2}}+\alpha \frac{d}{d x}-k^{2}\right)-i k v\right] H_{z}=0 .}
\end{gathered}
$$

It may occur (e.g., in the case of Saturn and Uranus) that multiple harmonic components $H_{m}$ of the magnetic field with differing wavenumbers $k_{m}$ are important. Each component, of course, satisfies the linear dynamo equation individually, but all must be included in the Taylor constraint. Because the integral in equation (6) is over a whole number of periods of each component, cross-terms of the form $\left(\nabla \times \boldsymbol{H}_{m}\right) \times \boldsymbol{H}_{m^{\prime}}$ $\left(m \neq m^{\prime}\right)$ vanish, and we can replace $\left\langle H_{x}\right\rangle^{2}$ by $\sum_{m}\left\langle H_{x m}\right\rangle^{2}$ in equation (9).

We nondimensionalize equations (9) and (10a) in terms of $\xi \equiv \alpha\left(x-x_{0}\right), h_{x} \equiv H_{x} p^{1 / 2} / H_{0} \quad\left(\right.$ where $\left.H_{0} \equiv \lim _{x \rightarrow \infty}\left\langle H_{x}\right\rangle\right)$, and $u=\alpha v / \omega$ (where $\omega \equiv \alpha \lim _{x \rightarrow \infty} v$ or $\omega \equiv \lim _{x \rightarrow \infty} d v / d x$, whichever is finite). The boundary conditions as $\xi \rightarrow \infty$ are thus $h_{x}=1$ (the phase of $h_{x}$ is arbitrary) and $u=1$ or $D u=1$, where $D \equiv \partial / \partial \xi$. There are then three dimensionless parameters in the problem: $K=k / \alpha \ll 1$ by assumption, the Chandrasekhar number $Q=H_{0}^{2} \delta / 4 \pi \rho v \alpha^{2} \lambda\left(x_{0}\right)$ (Chandrasekhar 1965, p. 7) and the magnetic Reynolds number $R_{m}=\omega / \alpha^{2} \lambda\left(x_{0}\right)$. The Chandrasekhar number expresses the importance of magnetic forces in determining $v$, and because of the variation of $\lambda$ we can always choose $x_{0}$ sufficiently deep that $Q=1$ there. The magnetic Reynolds number, on the other hand, expresses the importance of $v$ in modifying the magnetic field. Once we have chosen $x_{0}, R_{m}=4 \pi \rho v \omega / H_{0}^{2} \delta$, which may be of any magnitude. (In the case of multiple field harmonics of comparable strength, we make the obvious generalization of $H_{0}^{2}$ to $\sum_{m} H_{0 m}^{2}$ in these formulae.) Note that $H_{0}$ and $\omega$ are not predicted by the model but must be given as boundary conditions. Prediction of these quantities would require descriptions respectively of the regenerative dynamo and of the nonlinear interaction between convection and the zonal mean flow in the nonmagnetohydrodynamic limit (for which no satisfactory theory currently exists).

The appropriate nondimensionalization of the remaining field components is $h_{y}=H_{y} p^{1 / 2} / R_{m} H_{0}$ and $h_{z}=H_{z} p^{1 / 2}$. $\lim _{x \rightarrow \infty}\left\langle H_{z}\right\rangle$, with boundary conditions $h_{y}=0$ (no external "toroidal" field) and $h_{z}=1$ as $\xi \rightarrow \infty$. The full set of nondimensional equations (for $D^{2} \gg K D$ ) is:

$$
\begin{aligned}
\left(D^{2} \pm e^{-\xi}\left|h_{x}\right|^{2}\right) u & =0 \\
\left(D^{2}-i K R_{m} e^{-\xi} u\right) h_{x} & =0 \\
\left(D^{2}+D\right) h_{y} & =\left(-e^{-\xi} D u\right) h_{x}, \\
\left(D_{2}+D-i K R_{m} e^{-\xi}\right) h_{z} & =0 .
\end{aligned}
$$

In the limit $K R_{m}=0$, the above equations decouple, and an analytic solution may be obtained. Equations (11b) and (11d) reduce to $D^{2} h_{x}=\left(D^{2}+D\right) h_{z}=0$ so $h_{x}=h_{z}=1$ (we exclude a solution $h_{z} \sim e^{-\xi}$ due to "leakage" of currents from the dynamo region at great depth; its amplitude should be small). Equation (11a) thus becomes

$$
\left(D^{2} \mp e^{-\xi}\right) u=0
$$

or, with $\zeta=2 e^{-\xi / 2}$,

$$
\zeta^{2} \frac{d^{2} u}{d \zeta^{2}}+\zeta \frac{d u}{d \zeta} \mp \zeta^{2} u=0
$$

The solutions are $u \sim \mathscr{C}_{0}\left[(\mp 1)^{1 / 2} \zeta\right]$ (Abramowitz and Stegun 1965), where $\mathscr{C}_{0}$ is any Hankel or Bessel function of order zero. Converting to real argument and applying the boundary conditions [including $u<\infty$ as $\xi \rightarrow-\infty$, which excludes the solution proportional to $I_{0}(\zeta)$ for $\left.+v\right]$, we find three possible cases, illustrated in Figure 4 (solid curves):

$$
\begin{array}{ll}
u(\xi)=2 K_{0}\left(2 e^{-\xi / 2}\right), & \text { for }+v, D u(\infty)=1, \\
u(\xi)=J_{0}\left(2 e^{-\xi / 2}\right), & \text { for }-v, u(\infty)=1, \\
u(\xi)=c J_{0}\left(2 e^{-\xi / 2}\right)-\pi Y_{0}\left(2 e^{-\xi / 2}\right), & \text { for }-v, D u(\infty)=1,
\end{array}
$$
where $c$ is an arbitrary constant ( $c=0$ is illustrated). We refer to these solutions generically as $u=c \mathscr{F}_{0}\left(2 e^{-\xi / 2}\right)$. The exclusion of the fourth possibility [which has $D u(\infty)=0$ ] means that the condition of zero viscous stress at the exterior cannot be met for a positive eddy viscosity. For $+v$, a flux of energy and momentum from the outside is required to sustain the motion against viscous and Ohmic losses. In contrast, $-v$ permits "viscous" extraction of convective energy to balance Ohmic losses so that an energy/momentum input is not needed (though it can be accommodated). Indeed, the simplest descrip-

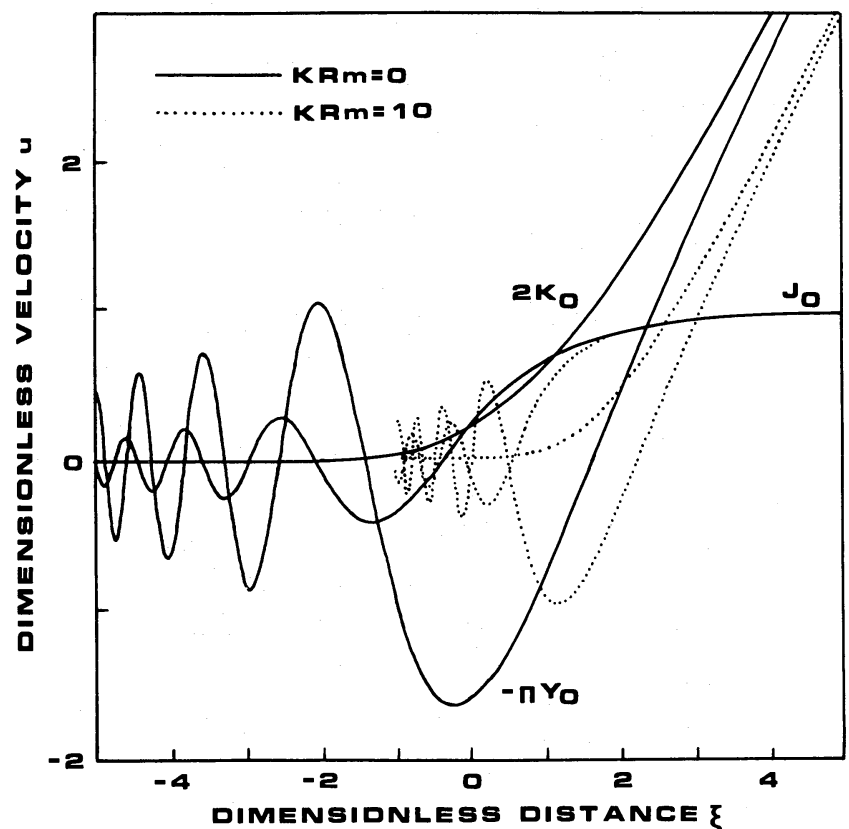

FIG. 4.-Dimensionless solutions for the zonal velocity. For $K R_{m}=0$ (solid curves), three analytic solutions (eq. [14]) are shown: for positive eddy viscosity one monotonic solution $2 K_{0}(\zeta)$, where $\zeta=2 \exp (-\zeta / 2)$; and for negative viscosity two oscillatory solutions $J_{0}(\zeta)$ and $-\pi Y_{0}(\zeta)$. For $K R_{m}=10$ (dotted curves) three numerical solutions are shown, similar in character but displaced to the right because of the inward amplification of the magnetic field $h_{x}$. 
tion of the energy supply needed for the solution (14a) is a region (outside the portion of the planet modeled) of negative eddy viscosity. For these reasons we consider the torque-free solution (14b) to be the one of greatest relevance and importance.

To complete the solution when $K R_{m}=0$, we note that equation (11c), $\left(D^{2}+D\right) h_{y}=e^{-\xi} D u$, may be integrated twice (again, we exclude an exponentially decaying "leakage" field by choosing the constant $u_{0}=0$ ), to give

$$
\begin{aligned}
h_{y}(\xi) & =\int_{\xi}^{\infty} e^{-\xi^{\prime}}\left[u\left(\xi^{\prime}\right)-u_{0}\right] d \xi^{\prime} \\
& =\frac{1}{2} \int_{0}^{2 e^{-\xi / 2}} \zeta c \mathscr{F}_{0}(\zeta) d \zeta \\
& =c e^{-\xi / 2 \mathscr{F}_{1}\left(2 \mathrm{e}^{-\xi / 2}\right) .}
\end{aligned}
$$

Figure 5 illustrates the three field components for the velocity solution $u=J_{0}\left(2 e^{-\xi / 2}\right)$.

From our solutions, one can calculate the Ohmic dissipation per unit volume $E_{\text {Ohmic }}=1 / \sigma\langle(c / 4 \pi \nabla \times H)\rangle^{2}$ and viscous dissipation (or energy release $\dot{E}_{\text {visc }}= \pm \rho v(d v / d x)^{2}$, averaged over one period in $y$. Defining $P=\rho v \omega^{2}$ and making use of $Q=1$, we find

$$
\begin{aligned}
\dot{E}_{\text {Ohmic }} & =P\left[2 e^{-\xi / 2} \frac{c}{2} \mathscr{F}_{0}\left(2 e^{-\xi / 2}\right)\right]^{2}, \\
\dot{E}_{\text {visc }} & = \pm P\left[2 e^{-\xi / 2} \frac{c}{2} \mathscr{F}_{1}\left(2 e^{-\xi / 2}\right)\right]^{2},
\end{aligned}
$$

illustrated in Figure 6 for the $2 K_{0}$ and $J_{0}$ solutions. For $+v$, both functions are positive and decay double-exponentially as

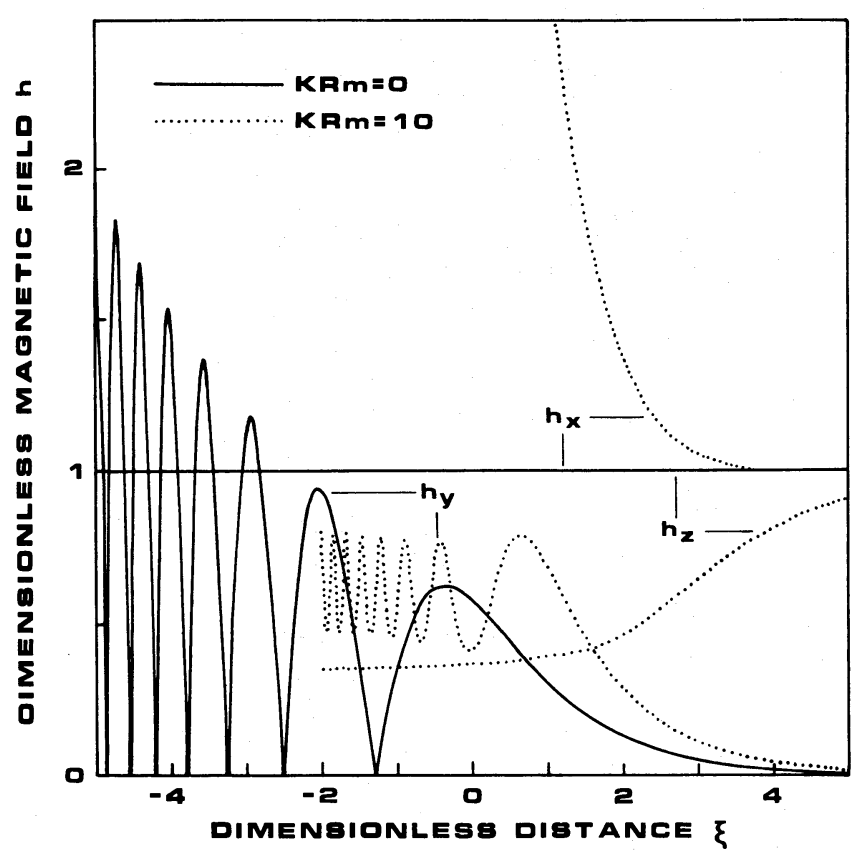

FIG. 5.-Dimensionless solutions for the magnetic field, corresponding to the $J_{0}$ analytic and $J_{0}$-like velocity solutions of Fig. 4. For $K R_{m}=0$ (solid curves), the "radial" field $h_{x}$ and "vertical" field $h_{z}$ are unaffected by the motion, but a substantial " toroidal" field $h_{y}$ is induced. For $K R_{m}=10$ (dotted curve), $h_{x}$ is inward-amplified and $h$ inward-attentuated. These field components can be thought of as the first term in an expansion about the equatorial plane.

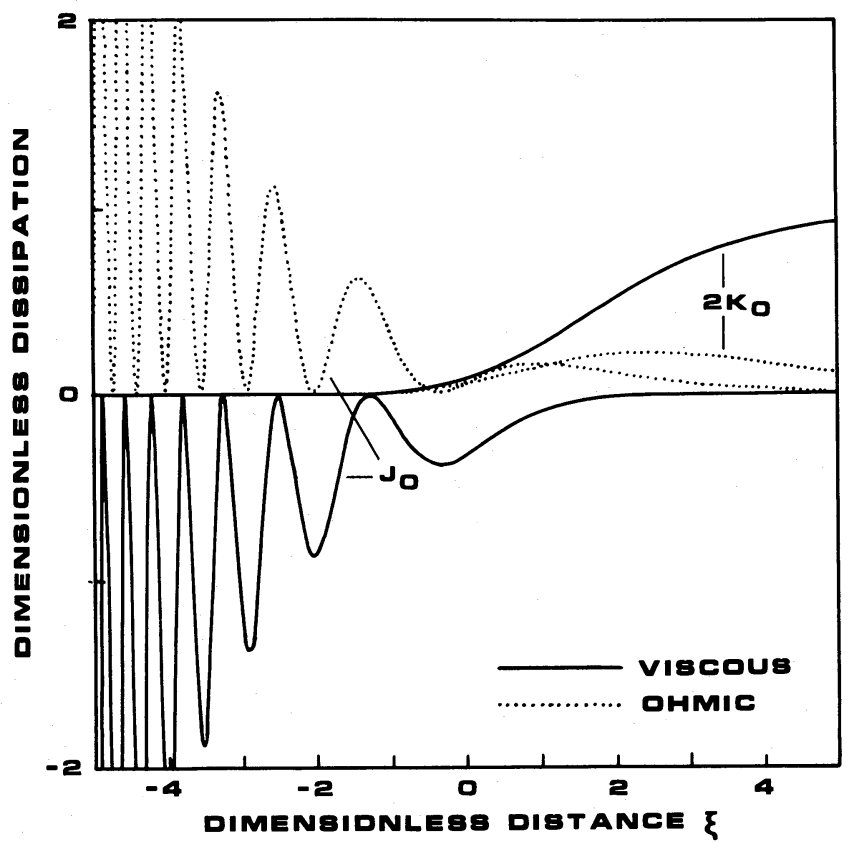

FIG. 6.-Dimensionless viscous (solid curve) and Ohmic (dotted curves) dissipation for two cases with $K R_{m}=0$. For positive viscosity (velocity solution $u=2 K_{0}(\zeta)$ in Fig. 4$)$, both terms are positive and vanish as $\xi \rightarrow-\infty$. For negative viscosity $\left[u=J_{0}(\zeta)\right]$, they are of opposite sign and divergent; "viscous" liberation of convective energy replenishes Ohmic losses. Similar behavior occurs for finite $K R_{m}$.

$\xi \rightarrow-\infty$; we find $\int_{-\infty}^{\infty} \dot{E}_{\text {Ohmic }} d x^{\prime} \approx 0.9980 P / \alpha$ and $\int_{-\infty}^{x} \dot{E}_{\text {visc }}$ $d x^{\prime} \approx(\alpha x-2.1450) P / \alpha$ as $x \rightarrow-\infty$. For $-v$, however, $\dot{E}_{\text {Ohmic }}$ and $\dot{E}_{\text {visc }}$ are oscillatory functions of opposite sign, and both diverge like exp $(-\alpha x / 2)$. Clearly, the assumption of constant eddy viscosity must break down at some depth where the mean flow attempts to extract more energy from convective eddies than is available.

For $K R_{m} \neq 0$, the coupled equations (11) must be solved numerically; but as Figure 4 shows, the character of the velocity solutions is not changed: for $+v$ there is a single " $K_{0}$-like" monotonic solution with $D u(\infty)=1$; for $-v$ there are both " $J_{0}$-like" $[u(\infty)=1]$ and " $Y_{0}$-like" $[D u(\infty)=1]$ oscillatory solutions. Figure 5 illustrates the behavior of the magnetic field for the torque-free " $J_{0}$-like" case (the other cases are qualitatively similar). We see that $\left|h_{x}\right|$ is inward-amplified to an asymptote which appears linear in $\xi$; in fact, as $\xi \rightarrow-\infty$, $D\left|h_{x}\right| \sim\left(K R_{m}\right)^{1 / 2}\left[0.1 \xi-\ln \left(2 K R_{m}\right)^{1 / 2}\right]$, so that $\left|h_{x}\right|$ is quadratic in $\xi$ (the asymptotic form of the phase of $h_{x}$ may also be obtained, but we do not discuss it here). Dimensionally, this implies that $d^{2}\left|H_{x}\right| / d x^{2}$ is fixed (for given $v, \omega$, etc.), and the external field value $H_{0}$ depends exponentially on $d\left|H_{x}\right| d x$ at given depth in the interior. This constitutes a boundary condition on the deep-seated region of dynamo action, in striking contrast to the conventional $d H_{x} / d x=0$ (for $D^{2} \gg K D$ ) in the absence of a conductivity gradient. A similar result is to be expected for the fully three-dimensional problem.

The "toroidal" field $h_{y}$ differs qualitatively from that for $K R_{m}=0$ only in that (for the case of $-v$ ) $\left|h_{y}\right|$ oscillates between positive bounds as $\xi \rightarrow-\infty$ rather than between zero and a diverging upper bound. In particular, the period of oscillation still decreases so rapidly as $\xi \rightarrow-\infty$ that $\dot{E}_{\text {Ohmic }}$ and $\dot{E}_{\text {visc }}$ diverge.

Unlike $h_{x}, h_{z}$ is inward-attenuated, reaching a limit of 
TABLE 1

Parameters for Equatorial Jet Models ${ }^{a}$

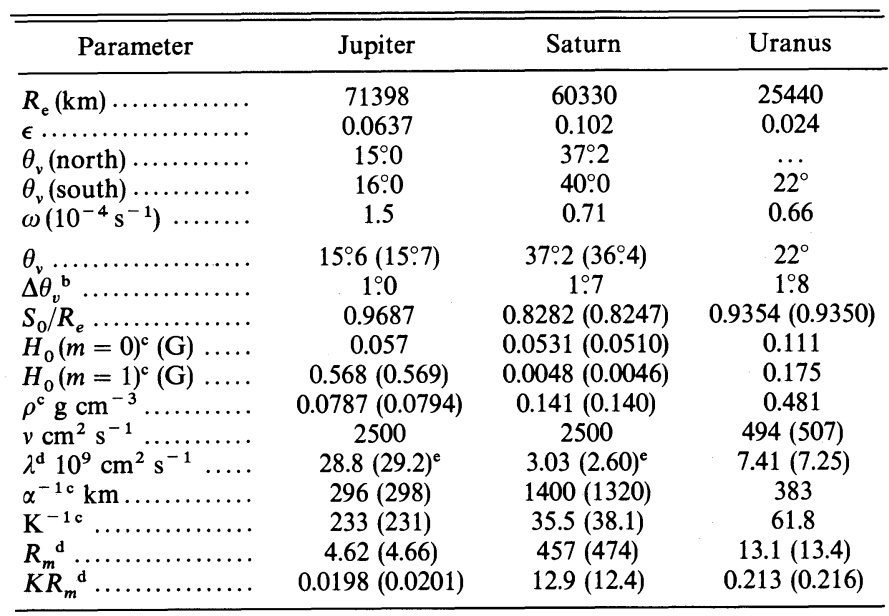

${ }^{a}$ Entries in parentheses are for the $K_{0}$-like model when different from those for the $J_{0}$-like model.

b Variation of $\theta_{v}$ when $\lambda$ is changed by $10^{1 / 2}$.

${ }^{c}$ Evaluated at $v=0$ location where model $\left(H_{0}, \alpha, K\right.$ constant $)$ is fitted to planet.

d Extrapolated to $Q=1$ with constant $\alpha$.

${ }^{\mathrm{e}}$ Upper limit on value of $\lambda(r)$, cf. Fig. 2.

$\left(K R_{m}\right)^{-1 / 2}$ as $\xi \rightarrow-\infty$ for large $K R_{m}$. This may, however, be a consequence of our assumption $\partial / \partial z=0$ rather than a reflection of the actual behavior of the field in three dimensions.

\section{APPLICATION TO JUPITER AND SATURN}

To apply the dimensional form of the model described in the last section to the equatorial jet of the giant planets, we use the eddy viscosity $v$ as a parameter to fit the observed jet width, then show that the required viscosity is consistent with the upper limit derived by Ingersoll and Pollard (1982). Of the remaining quantities, obtained from experiment or theory and listed in Table 1, a few deserve comment here.

Zonal velocity profiles based on Voyager imaging (Smith $e t$ al. 1979, 1982) were used to determine the jet width $\theta_{v}$ and shear amplitude $\omega$. For both the observations and the $J_{0}$-like models, $\theta_{v}$ was defined as the lowest latitude at which $v=0$ (or an average of the values in the two hemispheres if they differ). For the $K_{0}$-like models, in which $v \rightarrow 0$ only asymptotically, $\theta_{v}$ was defined by constructing a tangent to the dimensionless velocity $u(\xi)$ at $u=0.5$ and finding its zero crossing $\xi_{v}$, then mapping this location onto the surface of the planet in the usual way. With this definition $\theta_{v}(v)$ is the same for both types of model when $v$ is small. The distance from $Q=1$ to the zero crossing (or extrapolated zero crossing) of $v$ is a function of $K R_{m}$ and can be substantial: several times $\alpha^{-1}$. Thus, even to calculate a simple width parameter for the jet, we must solve the differential equations (11) in addition to finding the location $s_{0}$ at which $Q=1$. The shear amplitude was defined by $\omega=d v /\left.d s\right|_{\theta_{v}}$, a good approximation since the dimensionless shear $D u \approx 1$ near $u=0$.

The magnetic field strengths in Table 1 are rms radial fields in the equatorial plane at the locations where $v=0$, computed from the multipole expansions of Smith et al. (1976; model P11 312E) and Connerney et al. (1984; models $Z_{3}, \mathrm{P} 11 \mathrm{~A}$ as corrected in note in proof) according to the formulae

$$
\begin{aligned}
& H_{0, m=0}=\frac{3}{2}\left(R_{e} / s\right)^{4} g_{2}^{0}, H_{0, m=1} \\
& H_{0, m=1}=2^{-1 / 2}\left(R_{e} / s\right)^{3}\left[\left(g_{1}^{1}\right)^{2}+\left(h_{1}^{1}\right)^{2}\right]^{1 / 2}
\end{aligned}
$$

The $m=1$ value for Saturn is in fact a crude upper limit based on a dipole tilt of $1^{\circ}$; the nonaxisymmetry of Saturn's external magnetic field now appears to lie below the threshold of detectability. For both planets, the larger field component is uncertain to $\sim \pm 10 \%$ and the smaller to $\sim \pm 100 \%$. In applying our models, we can ignore the Jovian $m=0$ field, which contributes $\lesssim 1 \%$ of the total Lorentz force; for Saturn, both field components may be dynamically important since the nonaxisymmetric term is inward-amplified.

The magnetic diffusivity $\lambda$ was discussed at length above. The density $\rho$ as a function of depth was estimated using a constant gravitational acceleration and a polytropic equation of state for cosmic-composition gas (Stevenson 1982) in the equation of hydrostatic equilibrium.

Figure 7 shows the dependence of jet width on eddy viscosity obtained with these assumptions, and in particular the best-fit models whose parameters appear in Table 1 . We see that with the nominal magnetic diffusivity, $\bar{\theta}_{v}=15^{\circ} .5$ is obtained for Jupiter with $v$ only slightly in excess of the Ingersoll and Pollard (1982) limit based on the convective energy available for dissipation. For Saturn, $\theta_{v}=38^{\circ} .6$ cannot quite be attained (essentially because of the decrease of $\alpha$ with depth), but for reasonable $v, \theta_{v}$ is only a few degrees smaller. An increase of $\lambda$ by $10^{1 / 2}$ to the upper limit of our estimated uncertainty (indicated by shading) allows us to fit the equatorial jets of both planets with $v \approx 2500 \mathrm{~cm}^{2} \mathrm{~s}^{-1}$.

Figures 8 and 9 illustrate $J_{0}$-like and $K_{0}$-like models of the zonal velocity $v(\theta)$ computed with $v=2500 \mathrm{~cm}^{2} \mathrm{~s}^{-1}$ and $\lambda$ increased to the limit of uncertainty, superposed on the Voyager data (Smith et al. 1979, 1982). Attention should be restricted to $|\theta| \sim \theta_{v}$, since the models describe neither the possibility of "broken" Taylor columns at higher latitudes nor the complex interaction between convection and differential rotation that leads to the fine structure at lower latitudes. With these restrictions, we see that the data do not discriminate between the two classes of model (e.g., the oscillatory behavior of the $J_{0}$-like solution does not account for the existence of jets with $|\theta|>\theta_{v}$. The presence of a "shoulder" in the observed velocity profile (at $v \approx 40 \mathrm{~m} \mathrm{~s}^{-1}$ for Jupiter and $v \approx 80 \mathrm{~m} \mathrm{~s}^{-1}$ for Saturn) similar to that in the $J_{0}$-like model is, however, intriguing. The strongest conclusion which can be drawn is that the physical mechanism responsible for the shoulder operates on a length scale comparable to $\alpha^{-1}$ in each planet $\left(\alpha^{-1}\right.$ differs more than fourfold in the two cases), since when we adjust the model shear $\omega$ to the data, the shoulder velocity is reproduced as well.

For the sake of completeness, we show in Figures 10 and 11 the model radial and azimuthal magnetic field components in the equatorial plane. An oscillatory toroidal field of substantial strength is indicated for both planets. The Jovian magnetic Reynolds number is small $\left(K R_{m} \approx 0.02\right)$, so that the radial field is almost constant (geometric attenuation is neglected). In contrast, the much weaker field on Saturn results in $K R_{m} \approx 12$. The axisymmetric radial field is unaffected, but the $m=1$ field is substantially enhanced in the interior, at least in the equatorial plane. We return in $\S \mathrm{VI}$ to the intriguing question of whether a similar shielding effect operating at higher latitudes as well may be responsible for the high degree of axisymmetry of the externally measured field.

To restate the important points of this section, the model of $\S$ III reproduces the widths of the equatorial jets of Jupiter and Saturn for choices of the eddy viscosity $v$ which are similar in magnitude and roughly in keeping with the constraint imposed by available convective energy (especially if the assumed mag- 


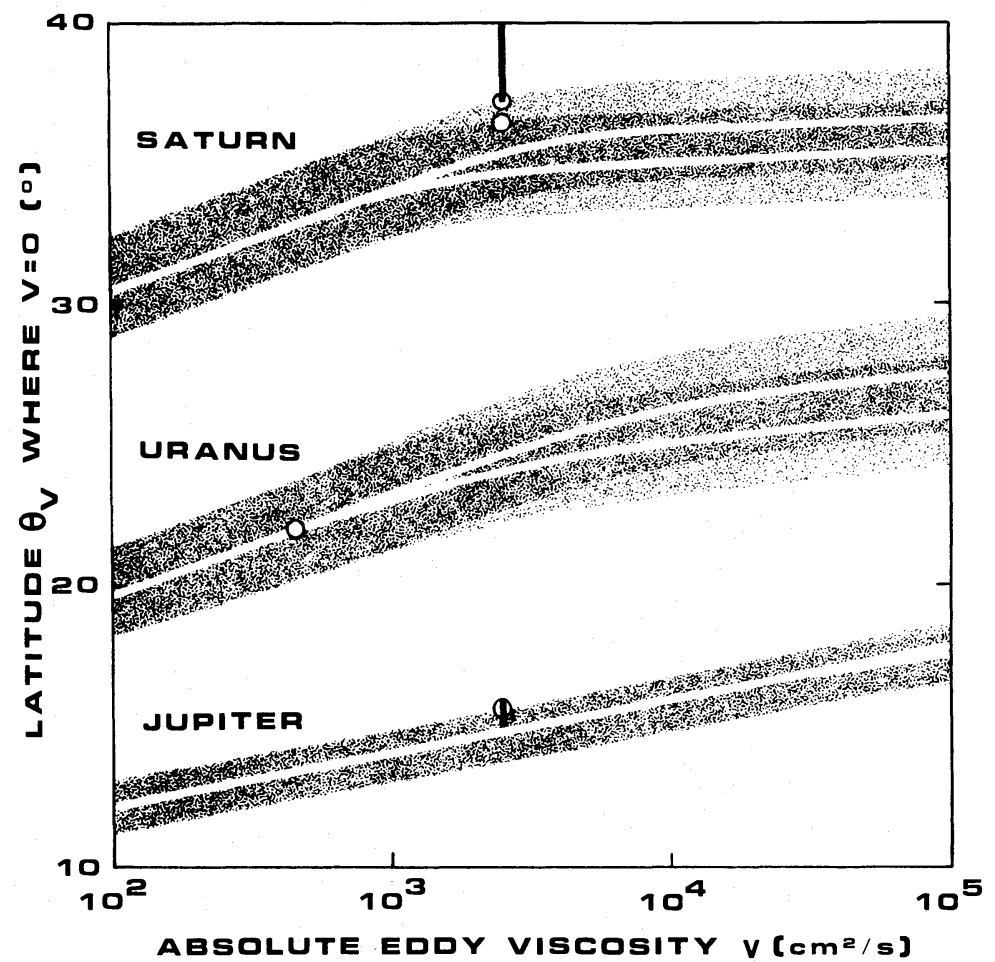

FIG. 7.-Width of the equatorial jet vs. eddy viscosity for $J_{0}$-like (upper of each pair of curves) and $K_{0}$-like models, with effect of $\pm 5 \mathrm{db}$ variation of $\lambda$ (cf. Fig. 2) indicated by shading. Vertical bars represent Voyager observations for two hemispheres, plotted at the upper limit $v=2500 \mathrm{~cm}^{2} \mathrm{~s}^{-1}$ based on available convective energy. Circles represent the models of Table 1 and Figs. 8-11.

netic diffusivity is increased within the limits of uncertainty). A large magnetic Reynolds number $K R_{m}$, and hence modification of the poloidal magnetic field, is indicated for Saturn but not for Jupiter.

\section{URANUS AND NEPTUNE}

Much less is known about these planets than about Jupiter and Saturn, but, as indicated in $\S$ II, the presence of abundant

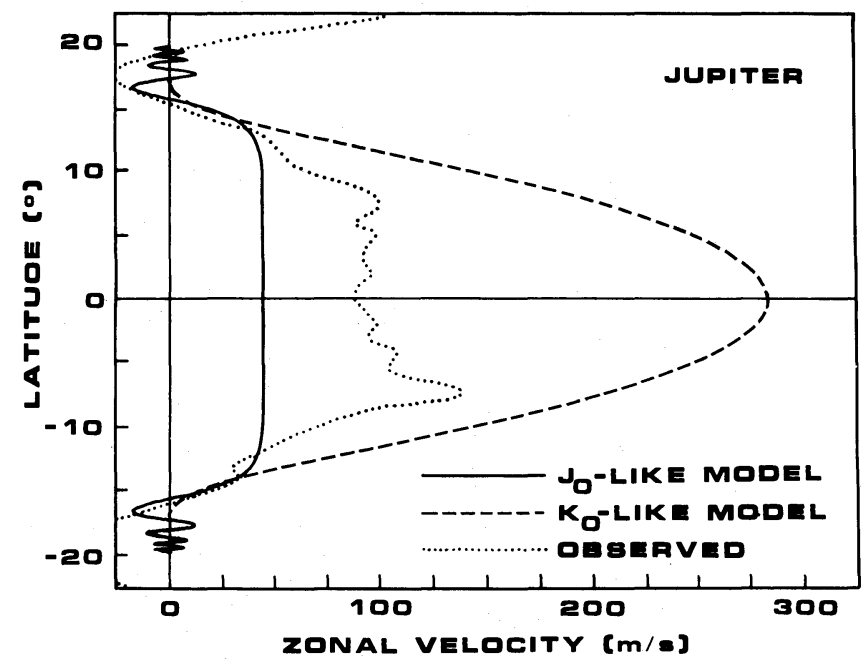

FIG. 8 water in their envelopes may lead to sustantial electrical conductivity. We show that Voyager 2 observations at Uranus are consistent with our equatorial jet model for a plausibly small choice of eddy viscosity, provided one assumes a two-layer internal structure, and we make the qualitative prediction of a wide equatorial jet on Neptune.

No equatorial jet has been directly observed on Uranus. Nonetheless, based on the success of our model in reproducing

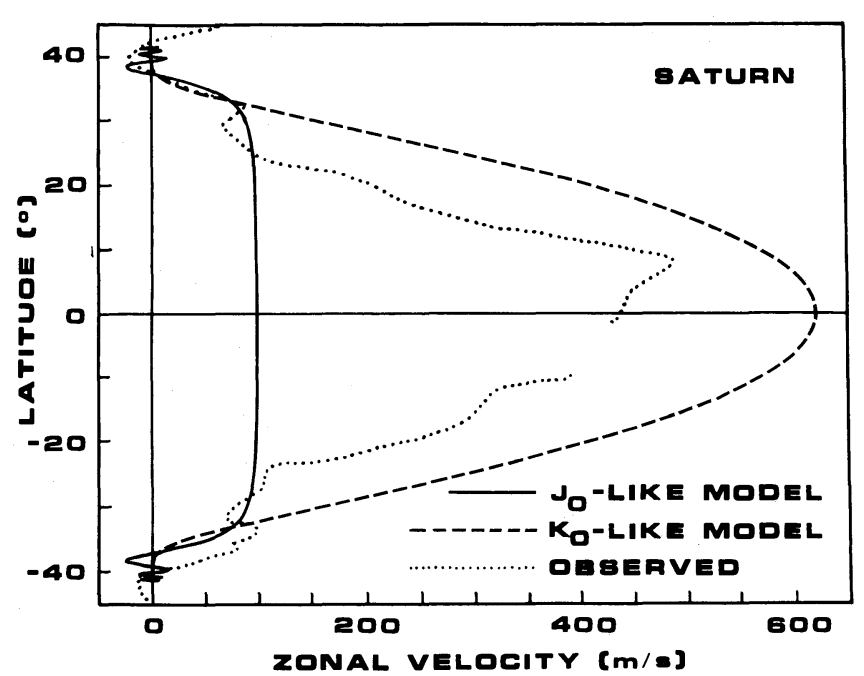

Fig. 9

FIG. 8.-Equatorial jet models for Jupiter. Best-fit models with negative (solid curve) and positive (dashed curve) viscosity are plotted along with Voyager data. Attention should be restricted to the regions near the zeros of velocity; the model does not describe the behavior of "broken "Taylor columns at high latitudes or the complex nonmagnetic effects responsible for the fine structure of the jet.

Fig. 9.-Same as Fig. 8, for Saturn 


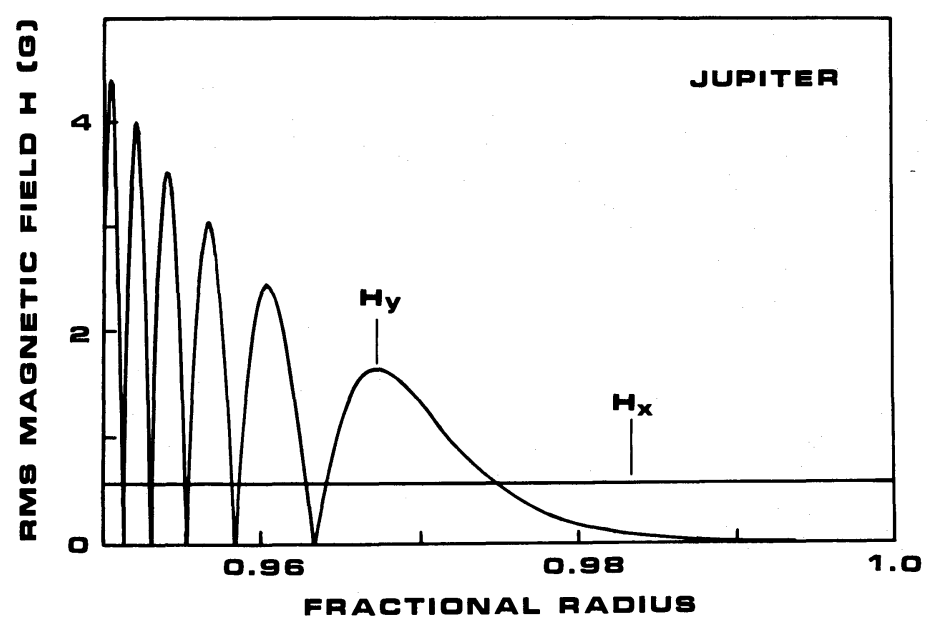

FIG. 10.-Magnetic field model for Jupiter. Radial $\left(H_{x}\right)$ and toroidal $\left(H_{y}\right)$ fields in the equatorial plane corresponding to the $J_{0}$-like velocity solution in Fig. 8 are shown. The quantity $H_{x}$ is near-constant because of the small magnetic Reynolds number (geometric attenuation has not been included).

the width of the Jovian and Saturnian jets even though it does not describe the "broken" Taylor columns which must exist at $|\theta|>\theta_{v}$, we make the case that parameters extrapolated from slightly higher latitudes may be used to characterize a possible Uranian equatorial jet. The Voyager 2 images (Smith et al. 1986) reveal seven cloud features at latitudes from $-25^{\circ}$ to $-70^{\circ}$, with rotation periods well described by the relation $\tau-\tau_{0}=0.081\left(\theta+22^{\circ}\right)+0.000455\left(\theta+22^{\circ}\right)^{2} \mathrm{hr}$, where $\tau_{0}=$ $17.24 \mathrm{hr}$ is the rotation period of the magnetic field. Three of these clouds lie between $-25^{\circ}$ and $-27^{\circ}$, where possible thermal wind corrections to their velocity are small (Hanel et al. 1986), lending confidence to the extrapolation to $\theta_{v}=22^{\circ}$, with $\omega=-6.6 \times 10^{-5} \mathrm{~s}^{-1}$ (i.e., a retrograde equatorial jet).

The Uranian magnetic field is unusual. We estimate significant $H_{0}$ for both $m=0$ and 1 based on the best-fit offset, tilted dipole (OTD) field model of Ness et al. (1986). The OTD parameters were first converted to planetocentric multipole moments according to the formulae of Smith et al. (1976, p. 799), then expressed as $H_{0}$ as in $\S$ IV.

We assume a two-layer model of the interior of Uranus. The "gas" layer of three-layer models is too cold to be conductive (cf. § II), so that the minimum hydromagnetically determined jet width occurs at $Q \geq 1$ at the top of the "ice" layer; for published models (Hubbard and MacFarlane 1980) this leads to $\theta_{v} \approx 45^{\circ}$, inconsistent with the Voyager data. We therefore model only the two-layer case in detail, converting the pressure-temperature dependence of $\mathrm{H}_{2} \mathrm{O}$ conductivity (Holtzapfel 1968) to depth dependence via the best-fit polytropic equation of state of Hubbard (1984) plus an approximate adiabatic temperature distribution, both in reasonable agreement with detailed models. As noted in $\S$ II, we adopt a tenfold suppression of the $\mathrm{H}_{2} \mathrm{O}$ conductivity by dissolved hydrogen-helium.

With these assumptions, we obtain the $\theta_{v}(v)$ curves shown in Figure 7. The inferred width of $22^{\circ}$ is obtained with an eddy viscosity of $\sim 500 \mathrm{~cm}^{2} \mathrm{~s}^{-1}$, substantially less than the value used for Jupiter and Saturn. This is in keeping with the limit on $v$ imposed by the availability of convective energy to be dissipated, since a rough upper limit on the internal heat flux of Uranus is an order of magnitude less than that of Saturn

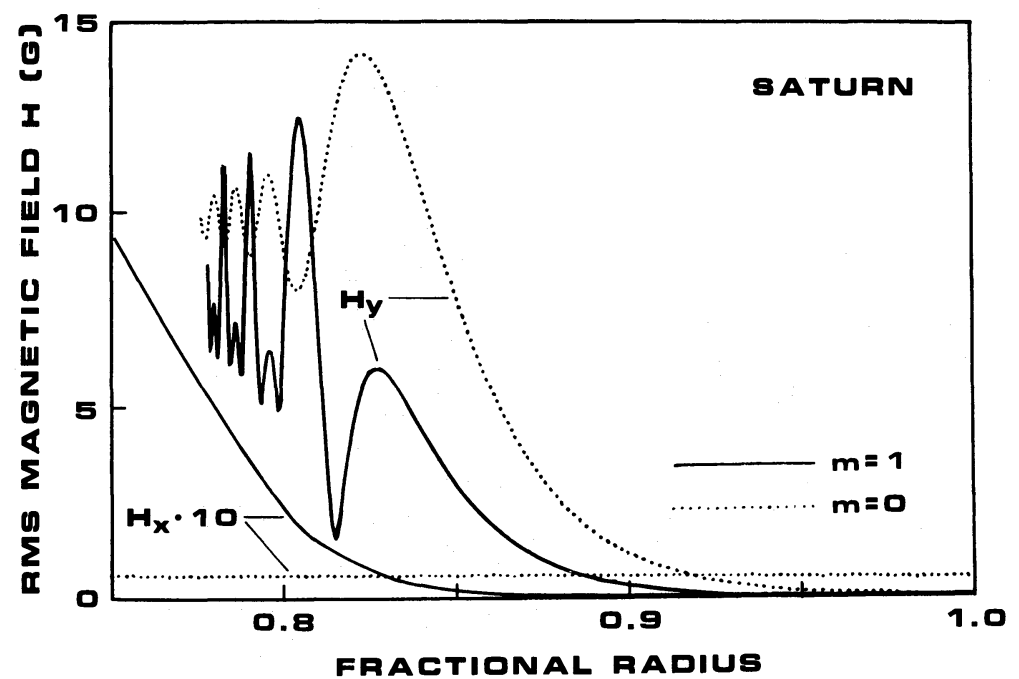

Fig. 11.-Same as Fig. 10, for Saturn. The nonaxisymmetric radial field (solid curve) is small in the exterior but strongly inward-amplified; the axisymmetric field (dotted curve) is constant, neglecting geometric attenuation. Each radial component induces a toroidal component (note different scales) of the same azimuthal wavenumber $m$. 
(Hubbard 1984). The magnetic Reynolds number obtained, $K R_{m} \approx 0.2$, is small, so that no axisymmetrization of the external field by the differentially rotating envelope is to be expected. This difference from Saturn results from the large inclination and offset of the Uranian dipole, which greatly increase $\sum_{m} H_{0 m}^{2}$, despite the comparable total magnetic moments of the two planets. Note that our estimates of $v$ and $K R_{m}$ for Uranus are in some sense upper limits, since the Voyager observations only constrain the width of the equatorial jet to be $\lesssim 22^{\circ}$.

Even less can be said about Neptune than about Uranus, but several factors point to a relatively wide Neptunian jet. First, Neptune is roughly similar to Uranus in temperature and in density (hence, presumably, composition), leading us to expect a similar conductivity structure in the two planets. Second, the measurably greater internal heat flux of Neptune (Hubbard 1984) leads to a correspondingly higher limit on the eddy viscosity. Finally, though the total moments of the Uranian and Neptunian magnetic fields might naively be expected to be similar (Hill and Michael 1975), the relevant magnetic field measure $H_{0}$ could be much less if Neptune does not share Uranus's high magnetic inclination and offset, which may be a consequence of its large obliquity (D. J. Stevenson, in preparation). The latter two effects both work to locate the $Q=1$ surface deeper in the planet, and hence to broaden the prediced equatorial jet.

\section{DISCUSSION}

The one-dimensional hydromagnetic model presented in the previous sections may be considered a success within the limits of its intended applicability: for plausible values of the diffusivities $\lambda$ and $v$, it reproduces the observed widths of the equatorial jets of three very different planets. As such it lends credence both to the hypothesis of (fairly) deep zonal flows and to the asserted importance of hydromagnetic effects outside the cores of the giant planets. Should one wish, however, to look beyond this single-parameter description to the details of the zonal flow, a number of problems arise which need clarification. Most are attributable (directly or indirectly) to the fact that the model is in essence an expansion about the equatorial plane on an assumed perfectly rigid Taylor column. Subsidiary problems arise from condensing all the physics of turbulent flow into the single parameter $v$.

An example of the latter class of problems is the failure of our model to duplicate the structure of the zonal velocity profile at the lowest latitudes. Inasmuch as the flow there is purely hydrodynamic, rather than hydromagnetic, the problem is far outside the scope of this paper, and we will say no more on the subject. The breakdown of the model at high latitudes, on the other hand, is both crucial and instructive. Starting with ideally coherent Taylor cylinders, we predict that at high latitudes the zonal wind will either vanish or oscillate on an everdecreasing length scale, far shorter than that which is observed. (The latter case, for negative eddy viscosity, also leads to diverging dissipation. This flow can be removed by making $v$ a decreasing function of the shear $d v / d s$, such that $v[d v / d s]^{2}$ is bounded, but the problem of too-rapid oscillation remains.) We conclude from this that Taylor cylinders reaching the surface at high latitude must be "broken," either trivially in the shallow atmosphere or at depth. In addition, in order for our model to work as well as it does at predicting the jet width $\theta_{v}$, this breaking must first occur at or slightly above that latitude. Our experience with the present model suggests that the flow is hydrodynamic and independent of axial coordinate $z$ outside a roughly spherical surface on which $Q=1$; and hydromagnetic, $z$-dependent, and possibly much slower inside that surface. The two-dimensional (if we assume $H \sim e^{i m \phi}$ ) problem of matching the inner flow to the outer remains as a challenging unsolved problem. A boundary layer analysis (i.e., neglecting horizontal derivatives) indicates that differential rotation on length scale much greater than $\alpha^{-1} \approx 10^{3} \mathrm{~km}$ could penetrate to the metallic core. Unfortunately, the observed jets on Jupiter and Saturn have widths comparable to $\alpha^{-1}$, making their analysis more difficult.

Knowledge of the dynamics of interrupted Taylor columns is also needed to answer the questions: What is the appropriate measure $H_{0}$ of the nonaxisymmetric field to use in the equatorial jet model when $K R_{m}$ is large? As a corollary, can the near spin-axisymmetry of Saturn's magnetic field be attributed to the zonal flow? For Jupiter and Uranus, this question does not arise. It is self-consistent to assume that the nonaxisymmetric field in the equatorial plane is unattenuated (hence given by the appropriate multipole component of the external field), since this leads to a flow model with $K R_{m} \lesssim 1$ at the $Q=1$ level. The situation for Saturn is more puzzling, since the radial field in the equatorial plane is strongly outward-attenuated by the magnetic skin effect. Two limiting possibilities suggest themselves, with $H_{0}$ ranging from zero to the external multipole value. We describe these extreme cases, without being able to choose between them or their intermediates. [Fortunately, in Saturn $H_{0, m=0}$ is large enough that the predicted value of $\theta_{v}(v)$ is affected only slightly by this uncertainty.] First, if the flow at high latitudes does not share the axisymmetrizing property of the equatorial jet, the observed $Y_{1}^{1}$ (tilted dipole) component of the external field connects (via high latitudes) to the interior. In the equatorial plane, $H_{s}$ is then small in the interior and smaller still outside: we should assume $H_{0, m=1} \approx 0$ in our model.

It is possible, on the other hand, that the magnetic field in the deep interior of Saturn is less axisymmetric than the external field would suggest. In the absence of an understanding of the two-dimensional hydromagnetic problem, the following conceptual model is instructive. Project the Voyager zonal wind profile along spin-axis concentric cylinders to the depth where $Q=1$ and calculate $K R_{m}$ as a function of latitude on this surface. Now idealize the attenuation process as follows: for some (scalar) quantity $\Phi$ with $Y_{1}^{1}$ angular dependence inside $Q=1$, let its value outside $Q=1$ be undiminished if $K R_{m}$ exceeds some threshold $K R_{m}^{*}$, but attenuated to zero locally if $K R_{m}>K R_{m}^{*}$. Expanding the exterior function in spherical harmonics then yields an overall attenuation factor for the $Y_{1}^{1}$ component of $\Phi$ from interior to exterior. Numerically, one obtains tenfold attenuation for $K R_{m}^{*} \approx 1.6$, a reasonable threshold based on our experience in the equatorial plane. This would seem to say that deep inside Saturn (but outside the dynamo region) the tilt of the dipole field could be $\mathcal{O}\left(10^{\circ}\right)$, comparable to that of Jupiter (and Earth). Though satisfying to the extent that the intrinsic axisymmetry of the Saturnian dynamo neet not be exceptional, this result is puzzling. Why would Jupiter and Saturn, with apparently similar fields below $Q=1$, experience such different amounts of axisymmetrization? A partial answer is that (based on our model) the shielding effect of the zonal flow leads to large field gradients as well as large fields in the interior. If the preceding arguments are correct, then the Saturnian dynamo is unexceptional in its dipole tilt-but exceptional in the richness of its higher multi- 
pole spectrum (corresponding to strong radial gradients of $H$ ). One wonders what has been gained.

It should be clear by now that the present work calls out for an investigation of the hydromagnetic flow of a planetary envelope in two dimensions. The encouraging results of our simple model, meanwhile, will be subject to further testing and refinement by the Voyager 2 encounter with Neptune in 1989 (which, hopefully, will increase our collection of equatorial jets by $33 \%$ ) and by direct observation of band-gap closure in hydrogen at high pressure.

This research was supported by NASA grant NAGW-185.

\section{REFERENCES}

Abramowitz, M., and Stegun, I. A. 1965, Handbook of Mathematical Functions (New York: Dover), p. 362

Allison, M., and Stone, P. H. 1983, Icarus, 54, 296

Ashcroft, N. W., and Mermin, N. D. 1976, Solid State Physics (New York: Holt, Rinehart, \& Winston), pp. 572-575.

Busse, F. H. 1976, Icarus, 29, 255.

1983, Geophys. Ap. Fluid Dyn., 23, 153.

Chandrasekhar, S. 1965. Hydrodynamic and Hydromagnetic Stability (New York: Dover)

Chapman, S., and Cowling, T. G. 1952, The Mathematical Theory of Nonuniform Gases (Cambridge: Cambridge University Press), p. 321.

Connerney, E. P., et al. 1984, in Saturn, ed. T. Gehrels and M. Matthews (Tucson: University of Arizona Press), p. 354.

Conrath, B. J., and Gierasch, P. J. 1984, Icarus, 57, 184.

Drobyshevskii, E. M. 1979a, Soviet Astr., 23, 334.

- 1979b, Soviet Astr., 23, 598.

Friedli, C., and Ashcroft, N. W. 1977, Phys. Rev., 16B, 662.

Goettel, K. A., Moss, W. C., Reichlin, R., and Martin, S. 1986, EoS, Trans. A.G.U., 67, 565

Hanel, R., et al., 1986, Science, 223, 70

Hide, R. 1965, in Magnetism and the Cosmos, ed. W. R. Hindmarsh, F. J. Lowes, P. H. Roberts, and S. K. Runcorn (Edinburgh: Oliver \& Boyd), p. 378

Hide, R., and Malin, S. R. C. 1979, Nature, 280, 42.

Hill, T. W. and Michel, F. C. 1975, Rev. Geophys. Space Phys., 13, 967.

Holtzapfel, W. B. 1968, J. Chem. Phys., 50, 4424

Hubbard, W. B. 1984, in Uranus and Neptune, ed. J. T. Bergstralh (Washington DC: NASA CP-2330), p. 291.

Randolph L. Kirk and David J. Stevenson: Division of Geological and Planetary Sciences, 170-25, California Institute of Technology, Pasadena, CA 91125
Hubbard, W. B., and Horedt, G. P. 1983, Icarus, 54, 456.

Hubbard, W. B., and Stevenson, D. J. 1984, in Saturn, ed. T. Gehrels and M. S. Matthews (Tucson: University of Arizona Press), p. 47.

Ingersoll, A. P., and Miller, R. L. 1986, Icarus, 65, 370.

Ingersoll, A. P., and Pollard, D. 1982, Icarus, 52, 62

Mao, H. K., Bell, P. M., and Hemley, R. J. 1985, Phys. Rev. Letters, $55,99$.

Min, B. I., Jansen, H. J. F., and Freeman, A. J. 1986, Phys. Rev., 33B, 6383.

Moffatt, H. K. 1978, Magnetic Field Generation in Electrically Conducting Fluids (Cambridge: Cambridge University Press), p. 298.

Mott, N. 1971, Phil. Mag., 24, 1.

Ness, N. F., et al. 1986, Science, 233, 85

Ross, M. 1985, Rept. Progr. Phys., 48, 1

Ross, M., Graboske, H. C., Jr., and Nellis, W. J. 1981, Phil. Trans. Roy. Soc. London, A, 303, 303.

Smith, B. A., et al. 1979 , Science, 206, 927. 1982, Science, 215,504 1986, Science, 233, 43.

Smith, E. J., et al. 1976, in Jupiter, ed. T. Gehrels (Tucson: University of Arizona Press), p. 788

Smoluchowski, R. 1972, Phys. Earth Planet. Int., 6, 48.

Stevenson, D. J. 1982, Ann. Rev. Earth Planet. Sci., 10, 257.

Stevenson, D. J., and Salpeter, E. E. 1976, in Jupiter, ed. T. Gehrels (Tucson: University of Arizona Press), p. 85.

Taylor, J. B. 1963, Proc. Roy. Soc. London A, 274, 274

Xu, J. A., Mao, H. K., and Bell, P. M. 1986, Science, 232, 1404.
Hubbard, W. B., and MacFarlane, J. J. 1980, J. Geophys. Res. 85, 225.

. 1975, Ap. J. (Letters), 200, L119. 